\title{
Network-based gene function inference method to predict optimal gene functions associated with fetal growth restriction
}

\author{
KE-JUN YE ${ }^{1}$, JIE DAI $^{1}$, LING-YUN LIU ${ }^{2}$ and MENG-JIA PENG ${ }^{1}$ \\ Departments of ${ }^{1}$ Gynaecology and Obstetrics and ${ }^{2}$ Clinical Laboratory, \\ Ruian People's Hospital, Ruian, Zhejiang 325200, P.R. China
}

Received January 4, 2017; Accepted July 21, 2017

DOI: $10.3892 / \mathrm{mmr} .2018 .9232$

\begin{abstract}
The guilt by association (GBA) principle has been widely used to predict gene functions, and a network-based approach may enhance the confidence and stability of the analysis compared with focusing on individual genes. Fetal growth restriction (FGR), is the second primary cause of perinatal mortality. Therefore, the present study aimed to predict the optimal gene functions for FGR using a network-based GBA method. The method was comprised of four parts: Identification of differentially-expressed genes (DEGs) between patients with FGR and normal controls based on gene expression data; construction of a co-expression network (CEN) dependent on DEGs, using the Spearman correlation coefficient algorithm; collection of gene ontology (GO) data on the basis of a known confirmed database and DEGs; and prediction of optimal gene functions using the GBA algorithm, for which the area under the receiver operating characteristic curve (AUC) was obtained for each GO term. A total of 115 DEGs and 109 GO terms were obtained for subsequent analysis. All DEGs were mapped to the CEN and formed 6,555 edges. The results of GBA algorithm demonstrated that $78 \mathrm{GO}$ terms had a good classification performance with AUC $>0.5$. In particular, the AUC for 5 of the GO terms was $>0.7$, and these were defined as optimal gene functions, including defense response, immune system process, response to stress, cellular response to chemical stimulus and positive regulation of biological process. In conclusion, the results of the present study provided insights into the pathological mechanism underlying FGR, and provided potential biomarkers for early detection and targeted treatment of this disease. However, the interactions between the 5 GO terms remain unclear, and further studies are required.
\end{abstract}

Correspondence to: Dr Ke-Jun Ye, Department of Gynaecology and Obstetrics, Ruian People's Hospital, 108 Wansong Road, Ruian, Zhejiang 325200, P.R. China

E-mail: kejunye2017@163.com

Key words: fetal growth restriction, gene ontology, co-expression network, guilt by association, prediction

\section{Introduction}

Fetal growth restriction (FGR), the second primary cause of perinatal mortality, is a clinical entity that affects $5-10 \%$ of gestations (1). FGR has multiple heterogeneous causes, including maternal, fetal and placental factors (2). Effective treatments for FGR have not been proposed, apart from the interruption of pregnancy (3). Consequently, early diagnosis and prevention is of importance for patients with FGR, which may permit the etiological identification and adequate monitoring of fetal vitality, minimizing the risks associated with prematurity and intrauterine hypoxia $(1,4)$. Therefore, the identification of biological markers for the early diagnosis and detection of FGR is required, in order to elucidate the molecular mechanism underlying FGR.

At present, numerous diseases have been attributed to the differential expression of genes compared with normal controls [differentially expressed genes (DEGs)] (5). However, genes frequently do not function individually; rather, they interact with other genes. A network-based approach is able to extract informative and notable genes via biological molecular networks, including the co-expression network (CEN), rather than focusing on individual genes $(6,7)$. Providing that gene connections are based on guilt-determination, predictions of their gene functions may be conducted utilizing guilt-by-association (GBA) method (8). The GBA is a basic element for predicting gene function, and typically uses the interactions between any two genes for the purpose of investigation the role of novel genes in gene function categories.

Therefore, the present study took Gene Ontology (GO) annotations and gene expression data as study objectives, and integrated the network approach with the GBA method, termed the network-based gene function inference method, for the purpose of predicting the optimal gene functions for FGR. These gene functions may be potential biomarkers for the early detection and targeted treatment of FGR.

\section{Materials and methods}

Network-based gene function inference method. The network-based gene function inference method was comprised of four steps: i) Identifying DEGs between patients with FGR and normal controls using Limma based on gene expression data; ii) constructing the CEN dependent on DEGs using the 
Spearman correlation coefficient (SCC); iii) collecting GO data for FGR on the basis of a known confirmed database and DEGs; and iv) predicting gene functions using the GBA algorithm, for which the area under the receiver operating characteristic curve (AUC) was calculated for each GO term. An AUC of 0.5 represents classification at chance levels, while an AUC of 1.0 represents a perfect classification. In the gene function prediction literature, AUC $>0.7$ is considered to be optimal (9). Therefore, GO terms with AUC $>0.7$ were defined as optimal gene functions for patients with FGR in the present study.

Identifying DEGs. Gene expression data [GSE24129 (2)] for human FGR were downloaded from the Gene Expression Omnibus database (www.ncbi.nlm.nih.gov/geo) using the accession number. GSE24129 was deposited on an Affymetrix Human Gene 1.0 ST Array (Affymetrix; Thermo Fisher Scientific, Inc., Waltham, MA, USA), and comprised normotensive pregnancies with or without FGR. The 8 examples with FGR were attributed to the case group, whereas 8 cases without FGR were denoted as normal controls. In order to control the quality of GSE24129, standard pretreatments were performed, which included background correction, normalization, probe matching and summarization (10-12). Following conversion of pretreated data at the probe level into gene symbols and removal of duplications, 14,398 genes were obtained for FGR in total.

Subsequently, DEGs between the FGR samples and normal controls were identified using the Limma package (13). The lmFit function implemented in Limma was utilized to perform empirical Bayes statistics and false discovery rate calibration of the P-values on the data $(14,15)$. Only genes which met the thresholds of $\mathrm{P}<0.05$ and $\log _{2}$ Fold Changel $>2$ were defined as DEGs across FGR patients and normal controls.

Constructing CEN. Cytoscape is an open source software project for integrating biomolecular interactions with high-throughput expression data and other molecular states into a unified conceptual network (16). Therefore, the DEGs were inputted into the Cytoscape software to visualize the CEN. In order to further evaluate the cooperated strength for each interaction in the CEN, the SCC method was utilized (17). SCC is a measure of the correlation between two variables, giving a value between -1 and +1 inclusive. If the SCC analysis returned a positive value, this indicated a positive linear correlation between two genes; otherwise, a negative correlation was indicated. For an interaction between gene i and j, the absolute SCC value was denoted as its weight value. The SCC was computed as follows:

$$
S C C=\frac{1}{n-1} \sum_{k=1}^{n}\left(\frac{g(i, k)-\dot{g}(i)}{\sigma(i)}\right) \cdot\left(\frac{g(j, k)-\dot{g}(j)}{\sigma(j)}\right)
$$

Where $n$ was the number of samples in the gene expression data; $g(i, k)$ or $g(j, k)$ was the expression level of gene $i$ or $j$ in the sample $k$ under a specific condition; and $\bar{g}(i)$ or $\bar{g}(j)$ represented the mean expression level of gene $i$ or $j$.

Recruiting GO annotation data. In the present study, the GO annotations were recruited from the GO Consortium (geneontology.org) (18). There were 19,003 terms and 18,402 genes in total for human beings. Notably, only one category (biological process) of GO was selected to be the study objective. In subsequent steps, the GO structure was diffused, and filtered for GO terms on size ranging from 20 to 1,000 genes after excluding those inferred from electronic annotation, a range that generally gives stable performance $(8,9)$. In addition, to make ensure that the GO terms correlated closely to FGR, if a GO term had a number of DEGs <20, it was removed. Therefore, only GO terms including $\geq 20$ DEGs were reserved. A total of 109 GO terms involved in 115 DEGs remained to be used in the following analyses.

Predicting gene function. As mentioned above, the GBA method was employed to predict the important gene function in the progression of FGR. Taking the GO functional annotations, a multi-functionality score (MFS) was assigned to each gene $i$ in the CEN (8):

$$
\operatorname{MFS}(i)=\sum_{x \vee i \in G O_{x}} \frac{1}{\operatorname{Num}_{i_{x}} * \text { Num }_{\text {out }_{x}}}
$$

Where Num $_{i n x}$ was the number of genes within GO group $x$, whose weighting had the effect of giving contribution to a GO group; and Num outx was the number of genes outside the GO group $x$ in the CEN, whose weighting provided a corresponding weight to genes outside the GO group. Therefore, as the only gene outside a large GO group, the score of the only gene within a GO group was added to the score of another gene. Notably, weighting referred to the impact of measuring connectivity in a group through the number of contributions of the gene to that GO group. A 3-fold cross-validation was applied to determine an MFS ranked list score for genes as to how well they fitted with the known gene set, and computed the AUC values for assessing the classification performances between FGR samples and normal controls. To the best of our knowledge, AUC has been introduced as a better measure for evaluating the predictive ability of machine learning in support vector machine (SVM) models compared with assessing the clinical classification performance (19). Consequently, the AUC values for GO terms were obtained, and terms with AUC $>0.7$ were identified to be optimal gene functions.

\section{Results}

DEGs and GO terms. In the present study, a total of 14,398 genes were obtained from the gene expression data following standard preprocessing. Based on these genes, 115 DEGs between FGR patients and normal controls were identified using the Limma package under the thresholds of $\mathrm{P}<0.05$ and $\| \log _{2}$ FoldChangel $>2$. As presented in Table I, all DEGs were ranked in ascending order of their P-values and the regulation directions were labeled; 58 were upregulated and 57 were downregulated. The most significant 5 DEGs were transmembrane protein $136\left(\mathrm{P}=4.09 \times 10^{-5}\right.$; downregulated $)$, acid phosphatase, prostate $\left(\mathrm{P}=5.42 \times 10^{-5}\right.$; downregulated $)$, protein tyrosine phosphatase, non-receptor type $3\left(\mathrm{P}=6.05 \times 10^{-5}\right.$; downregulated $)$, thrombospondin $1\left(\mathrm{P}=7.68 \times 10^{-5}\right.$; upregulated $)$ 
Table I. Differentially-expressed genes for fetal growth restriction.

\begin{tabular}{|c|c|c|c|}
\hline & \\
\hline & & Gene & Direction \\
\hline Gene & Direction & & \\
\hline TMEM136 & Down & CTSV & Down \\
\hline$A C P P$ & Down & $C 1 Q A$ & $\mathrm{Up}$ \\
\hline PTPN3 & Down & BHLHE4O & $\mathrm{Up}$ \\
\hline THBS1 & Up & ZC2HC1A & Down \\
\hline KCNK17 & Down & $F A M 26 D$ & Down \\
\hline TCN2 & $\mathrm{Up}$ & SH3TC2 & Down \\
\hline EDN1 & Up & TIMP1 & Up \\
\hline NNAT & Up & SLC38A9 & Down \\
\hline ZNF429 & Down & $L R P 2$ & Down \\
\hline TMEM168 & Down & DSC3 & Down \\
\hline SLA & Up & $T G F B I$ & $\mathrm{Up}$ \\
\hline$F 5$ & Down & LGR5 & Down \\
\hline TNNT3 & Up & GALNT11 & Down \\
\hline $\mathrm{P} 3 \mathrm{H} 2$ & Down & SEL1L3 & Down \\
\hline CATSPERB & Down & OR4F16 & Down \\
\hline BTNL9 & $\mathrm{Up}$ & OR4F21 & Down \\
\hline NAALADL2 & Down & LAPTM5 & $\mathrm{Up}$ \\
\hline GPERI & Up & $M E T$ & Down \\
\hline RPS6KA6 & Down & $D U S P 1$ & Up \\
\hline$A P L N$ & Down & NPR3 & Up \\
\hline$P G A P 1$ & Down & $P L A 2 G 2 A$ & Down \\
\hline$C T G F$ & Up & CHI3L1 & $\mathrm{Up}$ \\
\hline DHCR24 & Down & $C R H$ & Up \\
\hline ClGALT1 & Down & $E R A P 2$ & Down \\
\hline SOD1 & Down & $C 1 Q B$ & $\mathrm{Up}$ \\
\hline FBN2 & Down & EXTL2 & Down \\
\hline HISTIHIT & Down & PSMB9 & $\mathrm{Up}$ \\
\hline ADGRA3 & Down & CXCL9 & $\mathrm{Up}$ \\
\hline SLC41A2 & Down & CLDN1 & Up \\
\hline$L O X$ & Up & IFI44L & Up \\
\hline$C C D C 125$ & Down & $L G A L S 13$ & Down \\
\hline$F A M 234 B$ & Down & $H L A-D Q A 1$ & Up \\
\hline$S L C 20 A 1$ & Down & CXCL10 & Up \\
\hline ACSL1 & Up & $T A P 1$ & $\mathrm{Up}$ \\
\hline PLAC1 & Down & BCL6 & $\mathrm{Up}$ \\
\hline CYR61 & Up & GBP5 & $\mathrm{Up}$ \\
\hline GSTA3 & Down & FPR3 & $\mathrm{Up}$ \\
\hline$L G A L S 9 B$ & Up & $H L A-D Q B 1$ & $\mathrm{Up}$ \\
\hline GABRA4 & Down & WNT2 & Down \\
\hline DFNA5 & Down & HTRAl & $\mathrm{Up}$ \\
\hline$Q P C T$ & Up & KRTAP26-1 & $\mathrm{Up}$ \\
\hline$D D X 60 L$ & Down & FSTL3 & $\mathrm{Up}$ \\
\hline$M S L 3 P 1$ & Down & SLAMF7 & $\mathrm{Up}$ \\
\hline$A B C G 2$ & Down & $H L A-D Q A 2$ & $\mathrm{Up}$ \\
\hline$A D G R L 3$ & Down & HTRA4 & Up \\
\hline$A L D H 7 A 1$ & Down & $C G B 2$ & $\mathrm{Up}$ \\
\hline$A G L$ & Down & SLC27A2 & Down \\
\hline$C D 68$ & Up & CCL8 & $\mathrm{Up}$ \\
\hline$T F D P 2$ & Down & $H L A-D P B 1$ & $\mathrm{Up}$ \\
\hline$L E P$ & Up & ANKRD22 & $\mathrm{Up}$ \\
\hline$V W F$ & Up & $C G B 3$ & $\mathrm{Up}$ \\
\hline
\end{tabular}

Table I. Continued. 
Table I. Continued.

\begin{tabular}{lc}
\hline Gene & Direction \\
\hline$C P$ & $\mathrm{Up}$ \\
$C G B 1$ & $\mathrm{Up}$ \\
$C G B 5$ & $\mathrm{Up}$ \\
$H L A-D M A$ & $\mathrm{Up}$ \\
$C G B 7$ & $\mathrm{Up}$ \\
$A L A S 2$ & $\mathrm{Up}$ \\
$A O C 1$ & $\mathrm{Down}$ \\
$F C G R 3 A$ & $\mathrm{Up}$ \\
$H L A-D R A$ & $\mathrm{Up}$ \\
$L P L$ & $\mathrm{Up}$ \\
$U S P 9 Y$ & $\mathrm{Down}$ \\
$L Y Z$ & $\mathrm{Up}$ \\
\hline
\end{tabular}

and potassium two pore domain channel subfamily $\mathrm{K}$ member 17 ( $\mathrm{P}=1.32 \times 10^{-4}$; downregulated).

In addition, 19,003 GO terms and 18,402 genes associated with the biological process category of GO were collected from the GO Consortium. By removing terms with gene sizes not in the range 20-1,000 and intersected DEGs <20, $109 \mathrm{GO}$ terms including 115 DEGs were reserved. In order to illustrate the details of the GO annotations more clearly, a DEG enriched in one term was assigned a value of 1; otherwise, the value for the DEG in the GO term $=0$. The results are presented in Fig. 1, in which the yellow squares refer to 0 and red squares refer to 1 .

$C E N$. For the purpose of further investigating the biological activities of DEGs, a CEN with 115 nodes and 6,555 interactions for FGR was visualized using Cytoscape (Fig. 2A), which indicated that all DEGs were mapped to the CEN. In particular, the topological degree for each node was calculated by the sum of the nodes to which it was connected directly, and the degree distribution is presented in Fig. 2B. It was observed that the degrees for a large number of DEGs ( $55 \%)$ ranged between 56 and 60 , and the trend was approximately normally-distributed. Specifically, ankyrin repeat domain 22 possessed the highest degree of 65. Apart from the number of connections for each node, the interaction strength is a parameter that has been used to evaluate interactions in the CEN. Consequently, a weight was attributed to each edge using SCC analysis (data not shown). The heatmap for weights in the $\mathrm{CEN}$ is presented in Fig. $2 \mathrm{C}$. In the figure, squares represent edges in the CEN. Darker squares indicate larger weight values. Notably, a clear linear correlation was revealed among interactions, suggesting that the $\mathrm{CEN}$ exhibited good network scale properties.

Optimal gene functions. Prediction of gene function was performed using the GBA method, based on the integration between GO terms and the CEN. For each gene in a GO term, the MFS was computed. A high MFS indicated the possibility of a more optimal gene function. Therefore, all genes were ranked in descending order of the MFS and 3-fold cross-validation was performed to calculate the AUC for the

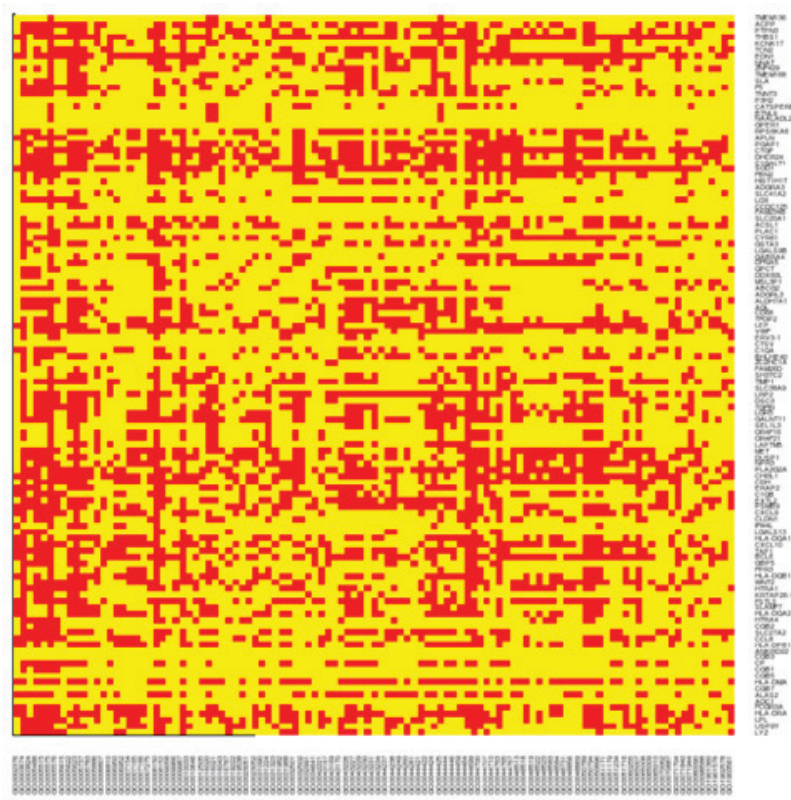

Figure 1. Functional annotation of DEGs in GO terms of fetal growth restriction. A total of 115 DEGs and 109 GO terms are presented. Red represents DEGs which were enriched in the GO term; yellow indicates that the DEG was not enriched in the GO term. DEG, differentially-expressed gene; GO, gene ontology.

GO terms, with the aim of classifying patients with FGR and normal controls. The AUC distribution among GO terms is illustrated in Fig. 3. The AUC for the majority of GO terms fell within the range $0.4-0.7$, particularly $0.6-0.65$. When AUC was used as a predictor of GO category membership, $78 \mathrm{GO}$ terms of $\mathrm{AUC}>0.5$ were obtained. It was noted that this single ranking of genes gave a mean AUC of 0.57 across all GO terms tested. In addition, 5 of the $78 \mathrm{GO}$ terms had an AUC >0.7 and were denoted as optimal gene functions (Table II): Defense response $(\mathrm{GO}: 0006952 ; \mathrm{AUC}=0.861)$, immune system process (GO:0002376; $\mathrm{AUC}=0.789)$, response to stress (GO:0006950; $\mathrm{AUC}=0.759)$, cellular response to chemical stimulus (GO:0070887; $\mathrm{AUC}=0.724)$ and positive regulation of biological process (GO:0048518; $\mathrm{AUC}=0.720$ ).

\section{Discussion}

Co-expression analysis dependent on networks has been used widely due to its good statistical confidence for individual connections, overlap with protein interactions, and mathematical convenience (20). In addition, the criterion in a CEN is generally divided into two types: Hard thresholding, which produces less robust results (21) and soft thresholding. Specifically, soft thresholding works well in network analysis (22) by combining greater sparsity with similarity to the original correlation matrix (23), for example in a weighted CEN. Pearson's correlation coefficient (PCC) is the most widely used measure for co-expression analysis. SCC is a nonparametric (distribution-free) rank statistical measure of a monotone association that is used when the distribution of data makes PCC undesirable or misleading (24). Therefore, in the present study, SCC was implemented to weight the CEN which was constructed dependent on DEGs for FGR, 
A

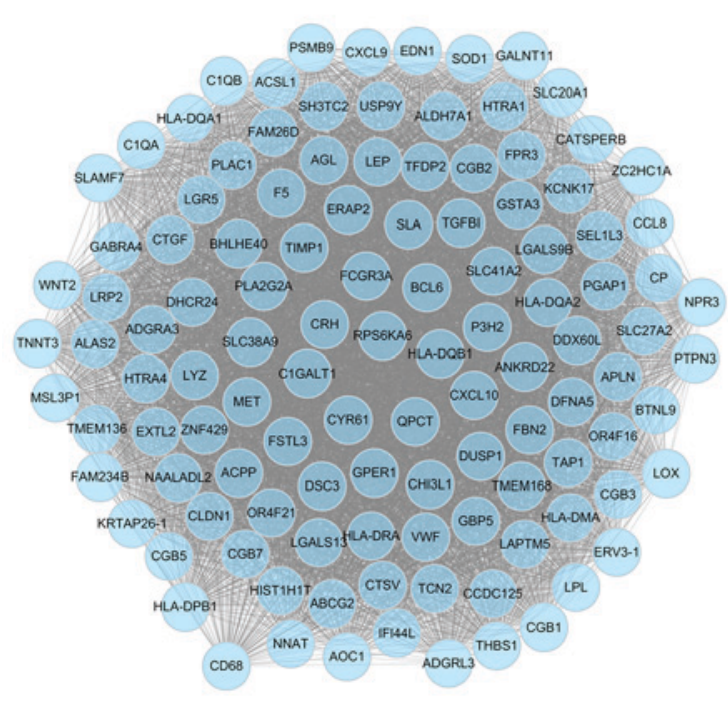

B

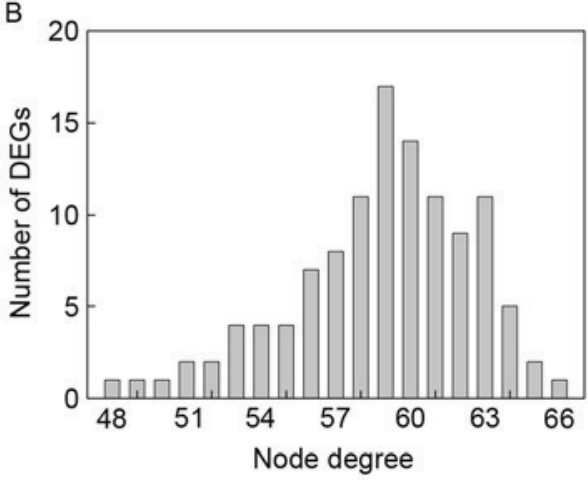

C

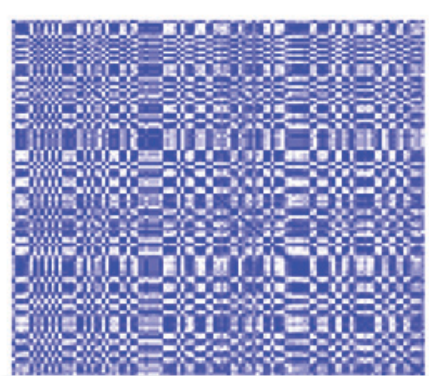

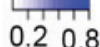

0.20 .8

Figure 2. Co-expression network for fetal growth restriction. (A) Diagram of the co-expression network. Nodes represent DEGs; edges represent interactions. (B) Node degree distribution of DEGs mapped to the co-expression network. (C) Weight distribution of edges in the co-expression network. The horizontal and vertical axes were DEGs mapped to the network, and the heatmap clarified the weight distribution for each edge. An apparent linear association was revealed. DEG, differentially-expression gene.

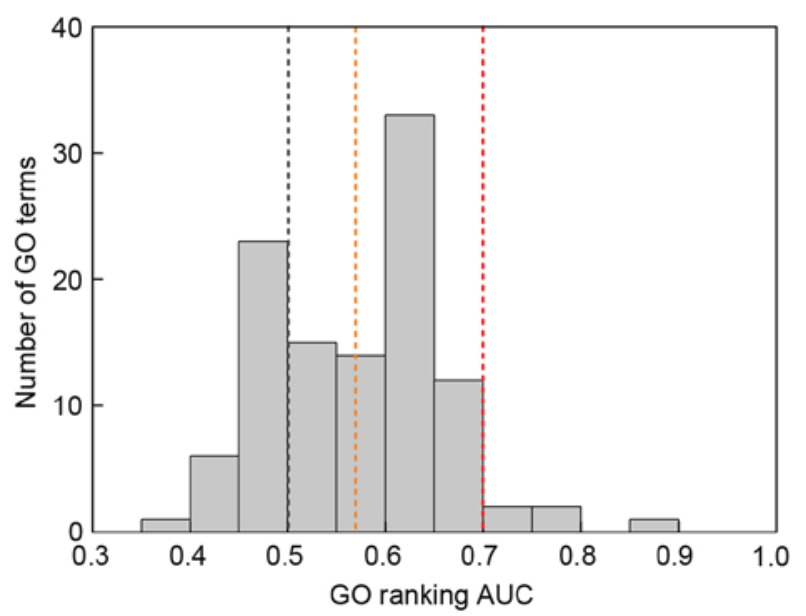

Figure 3. Gene function prediction performance using guilt by association analysis. The red line represents the threshold for optimal GO (AUC=0.7); the grey line indicates a GO performance considered to be good ( $\mathrm{AUC}=0.5$ ); the orange line represents the mean of all AUCs. AUC, area under the receiver operating characteristic curve; GO, gene ontology.

and its weight distribution suggested that the CEN had good scale network properties. There were 115 nodes and 6,555 interactions in the CEN, which was prepared for subsequent analysis.

Previously, various methods have been produced to expand the scale of the GBA to indirect connections, including weighting indirect connections by local topology, network propagation and topological overlap $(23,25,26)$. The majority of these methods refer to improvement over GBA between direct connections, although they tend to perform comparably and only slightly better than direct GBA (27). The present study integrated the GBA method with $\mathrm{CEN}$-associated analysis to further explore direct and indirect optimal gene functions for FGR, based on GO annotations and gene expression data. A network based-GBA or extended-GBA approach may facilitate the exhaustive examination of issues (due to being less subject to fine-tuning) compared with simple GBA. In the present study, an MFS was assigned to each gene enriched in the GO term. Ranking genes by AUC based on MFS was demonstrated to be a means of obtaining good performance from a gene function prediction algorithm, which validated the feasibility and confidence of the network-based GBA method. The results of the present study demonstrated that 78 GO terms had a good classification performance with an AUC $>0.5 ; 5$ of the GO terms had an AUC $>0.7$ and were defined as optimal gene functions, which included defense response, immune system process, response to stress, cellular response to chemical stimulus and positive regulation of biological process.

Specifically, defense response refers to reactions triggered in response to the presence of a foreign body or the occurrence of an injury, and results in restriction of damage to the organism attacked or prevention/recovery from the infection caused by the attack (28). Therefore, it is reasonable to infer that alterations of defense response caused by certain unexplained and unknown reasons in pregnancy may lead to the occurrence of FGR. In addition, immune system process includes any process involved in the development or functioning of the immune system, and is an organismal system which produces calibrated responses to potential internal or invasive threats (29). The immune system is a host defense system comprising a number of biological structures and processes within an organism that protect against disease, and disorders of the immune system 
Table II. GO terms with AUC $>0.5$.

\begin{tabular}{|c|c|c|c|}
\hline Ranking & ID & GO term & AUC \\
\hline 1 & GO:0006952 & Defense response & 0.861 \\
\hline 2 & GO:0002376 & Immune system process & 0.789 \\
\hline 3 & GO:0006950 & Response to stress & 0.759 \\
\hline 4 & GO:0070887 & Cellular response to chemical stimulus & 0.724 \\
\hline 5 & GO:0048518 & Positive regulation of biological process & 0.720 \\
\hline 6 & GO:0044459 & Plasma membrane part & 0.688 \\
\hline 7 & GO:0005615 & Extracellular space & 0.687 \\
\hline 8 & GO:0010033 & Response to organic substance & 0.685 \\
\hline 9 & GO:0048522 & Positive regulation of cellular process & 0.681 \\
\hline 10 & GO:0048583 & Regulation of response to stimulus & 0.681 \\
\hline 11 & GO:0050896 & Response to stimulus & 0.679 \\
\hline 12 & GO:0048584 & Positive regulation of response to stimulus & 0.678 \\
\hline 13 & GO:0044237 & Cellular metabolic process & 0.676 \\
\hline 14 & GO:0065009 & Regulation of molecular function & 0.675 \\
\hline 15 & GO:0007166 & Cell surface receptor linked signal transduction & 0.671 \\
\hline 16 & GO:0044249 & Cellular biosynthetic process & 0.666 \\
\hline 17 & GO:0042221 & Response to chemical & 0.652 \\
\hline 18 & GO:0050794 & Regulation of cellular process & 0.647 \\
\hline 19 & GO:0005488 & Binding & 0.646 \\
\hline 20 & GO:0032991 & Macromolecular complex & 0.644 \\
\hline 21 & GO:0043169 & Cation binding & 0.643 \\
\hline 22 & GO:0048523 & Negative regulation of cellular process & 0.640 \\
\hline 23 & GO: 1901576 & Organic substance biosynthetic process & 0.638 \\
\hline 24 & GO:0080090 & Regulation of primary metabolic process & 0.633 \\
\hline 25 & GO:0044421 & Extracellular region part & 0.633 \\
\hline 26 & GO:0031988 & Membrane-bounded vesicle & 0.633 \\
\hline 27 & GO:0031224 & Intrinsic component of membrane & 0.632 \\
\hline 28 & GO:0065007 & Biological regulation & 0.632 \\
\hline 29 & GO:0044700 & Single organism signaling & 0.630 \\
\hline 30 & GO:0065010 & Extracellular membrane-bounded organelle & 0.630 \\
\hline 31 & GO:0048519 & Negative regulation of biological process & 0.627 \\
\hline 32 & GO:0051716 & Cellular response to stimulus & 0.627 \\
\hline 33 & GO:0005886 & Plasma membrane & 0.626 \\
\hline 34 & GO:0005576 & Extracellular region & 0.625 \\
\hline 35 & GO:0003824 & Catalytic activity & 0.623 \\
\hline 36 & GO:0048869 & Cellular developmental process & 0.623 \\
\hline 37 & GO:0005783 & Endoplasmic reticulum & 0.622 \\
\hline 38 & GO:0031982 & Vesicle & 0.622 \\
\hline 39 & GO:0044260 & Cellular macromolecule metabolic process & 0.621 \\
\hline 40 & GO:0007154 & Cell communication & 0.619 \\
\hline 41 & GO:1903561 & Extracellular vesicle & 0.614 \\
\hline 42 & GO:0023051 & Regulation of signaling & 0.610 \\
\hline 43 & GO:0031323 & Regulation of cellular metabolic process & 0.608 \\
\hline 44 & GO:0060255 & Regulation of macromolecule metabolic process & 0.607 \\
\hline 45 & GO:0023052 & Signaling & 0.606 \\
\hline 46 & GO:0009058 & Biosynthetic process & 0.605 \\
\hline 47 & GO:0043234 & Protein complex & 0.603 \\
\hline 48 & GO:0044425 & Membrane part & 0.603 \\
\hline 49 & GO:0071840 & Cellular component organization or biogenesis & 0.601 \\
\hline 50 & GO:0043230 & Extracellular organelle & 0.600 \\
\hline 51 & GO:0043226 & Organelle & 0.599 \\
\hline 52 & GO:0051171 & Regulation of nitrogen compound metabolic process & 0.592 \\
\hline
\end{tabular}


Table II. Continued.

\begin{tabular}{|c|c|c|c|}
\hline Ranking & ID & GO term & AUC \\
\hline 53 & GO:0043227 & Membrane-bound organelle & 0.592 \\
\hline 54 & GO:0005515 & Protein binding & 0.590 \\
\hline 55 & GO:0008152 & Metabolic process & 0.589 \\
\hline 56 & GO:0044765 & Single-organism transport & 0.576 \\
\hline 57 & GO:0043167 & Ion binding & 0.573 \\
\hline 58 & GO:0065008 & Regulation of biological quality & 0.573 \\
\hline 59 & GO:0043229 & Intracellular organelle & 0.567 \\
\hline 60 & GO:0016021 & Integral component of membrane & 0.558 \\
\hline 61 & GO:0006810 & Transport & 0.558 \\
\hline 62 & GO:0051179 & Localization & 0.555 \\
\hline 63 & GO:0050789 & Regulation of biological process & 0.550 \\
\hline 64 & GO:0009966 & Regulation of signal transduction & 0.548 \\
\hline 65 & GO:0008150 & Biological process & 0.546 \\
\hline 66 & GO:0005623 & Cell & 0.544 \\
\hline 67 & GO:0071944 & Cell periphery & 0.536 \\
\hline 68 & GO:0019222 & Regulation of metabolic process & 0.535 \\
\hline 69 & GO:0043170 & Macromolecule metabolic process & 0.534 \\
\hline 70 & GO:0051234 & Establishment of localization & 0.532 \\
\hline 71 & GO:0007165 & Signal transduction & 0.527 \\
\hline 72 & GO: 1901360 & Organic cyclic compound metabolic process & 0.514 \\
\hline 73 & GO:0031090 & Organelle membrane & 0.512 \\
\hline 74 & GO:0016043 & Cellular component organization & 0.511 \\
\hline 75 & GO:0044710 & Single-organism metabolic process & 0.511 \\
\hline 76 & GO:0019538 & Protein metabolic process & 0.510 \\
\hline 77 & GO:0034641 & Cellular nitrogen compound metabolic process & 0.509 \\
\hline 78 & GO:0010646 & Regulation of cell communication & 0.507 \\
\hline
\end{tabular}

GO, gene ontology; AUC, area under the receiver operating characteristic curve.

may lead to autoimmune diseases, inflammatory diseases and cancer (30). It had been demonstrated that cytokines drive the innate immune response, and they are logical candidates for the disruption of fetal brain development (31). Therefore, immune system process was observed to be correlated to the progression of FGR. Regarding cellular response to chemical stimulus, this gene function comprises any process that results in a change in state or activity of a cell (e.g. movement, secretion, enzyme production or gene expression) as a result of a chemical stimulus (32). Therefore, pregnant women are recommended to be alert to the possibility of chemical stimuli within their food and water intake.

In conclusion, the present study identified 5 optimal gene functions in the process of FGR. The present findings may provide insights into the pathological mechanism underlying FGR, and provide potential biomarkers for the early detection and targeted treatment of this disease. However, the potential interactions between the $5 \mathrm{GO}$ terms remain to be elucidated in future studies.

\section{Acknowledgements}

Not applicable.

\section{Funding}

No funding was received.

\section{Availability of data and materials}

The datasets used and/or analyzed during the current study are available from the corresponding author on reasonable request.

\section{Authors' contributions}

KY made substantial contributions to the design of the present study and drafted the paper. JD conducted literature searching for the paper. LL and MP conducted data analysis and manuscript revised the manuscript. All authors read and approved the final manuscript.

\section{Ethics approval and consent to participate}

Not applicable.

\section{Patient consent for publication}

Not applicable. 


\section{Competing interests}

The authors declare that they have no competing interests.

\section{References}

1. Nardozza LM, Araujo Júnior E, Barbosa MM, Caetano AC, Lee DJ and Moron AF: Fetal growth restriction: Current knowledge to the general Obs/Gyn. Arch Gynecol Obstet 286: 1-13, 2012.

2. Nishizawa H, Ota S, Suzuki M, Kato T, Sekiya T, Kurahashi H and Udagawa Y: Comparative gene expression profiling of placentas from patients with severe pre-eclampsia and unexplained fetal growth restriction. Reprod Biol Endocrinol 9: 107, 2011.

3. Veiby G, Daltveit AK, Engelsen BA and Gilhus NE: Fetal growth restriction and birth defects with newer and older antiepileptic drugs during pregnancy. J Neurol 261: 579-588, 2014.

4. Figueras $\mathrm{F}$ and Gratacós E: Update on the diagnosis and classification of fetal growth restriction and proposal of a stage-based management protocol. Fetal Diagn Ther 36: 86-98, 2014.

5. Zhao J, Yang TH, Huang Y and Holme P: Ranking candidate disease genes from gene expression and protein interaction: A Katz-centrality based approach. PLoS One 6: e24306, 2011.

6. Liu ZP, Wang Y, Zhang XS and Chen L: Network-based analysis of complex diseases. IET Syst Biol 6: 22-23, 2012.

7. Chen L, Wang RS and Zhang XS: Reconstruction of Gene Regulatory Networks. In: Biomolecular Networks. John Wiley \& Sons, Inc, pp47-87, 2009.

8. Gillis $\mathrm{J}$ and Pavlidis P: The impact of multifunctional genes on 'guilt by association' analysis. PLoS One 6: e17258, 2011.

9. Gillis J and Pavlidis P: The role of indirect connections in gene networks in predicting function. Bioinformatics 27: 1860-1866, 2011.

10. Irizarry RA, Bolstad BM, Collin F, Cope LM, Hobbs B and Speed TP: Summaries of Affymetrix GeneChip probe level data Nucleic Acids Res 31: e15, 2003.

11. Bolstad BM, Irizarry RA, Astrand M and Speed TP: A comparison of normalization methods for high density oligonucleotide array data based on variance and bias. Bioinformatics 19: 185-193, 2003.

12. Bolstad B: affy: Built-in processing methods. Journal, 2013.

13. Ritchie ME, Phipson B, Wu D, Hu Y, Law CW, Shi W and Smyth GK: Limma powers differential expression analyses for RNA-sequencing and microarray studies. Nucleic Acids Res 43: e47, 2015 .

14. Datta S, Satten GA, Benos DJ, Xia J, Heslin MJ and Datta S: An empirical bayes adjustment to increase the sensitivity of detecting differentially expressed genes in microarray experiments. Bioinformatics 20: 235-242, 2004

15. Reiner A, Yekutieli D and Benjamini Y: Identifying differentially expressed genes using false discovery rate controlling procedures. Bioinformatics 19: 368-375, 2003.
16. Shannon P, Markiel A, Ozier O, Baliga NS, Wang JT, Ramage D, Amin N, Schwikowski B and Ideker T: Cytoscape: A software environment for integrated models of biomolecular interaction networks. Genome Res 13: 2498-2504, 2003.

17. Szmidt E and Kacprzyk J: The Spearman rank correlation coefficient between intuitionistic fuzzy sets. Journal: 276-280, 2010.

18. Gene Ontology Consortium: Gene ontology consortium: Going forward. Nucleic Acids Res 43 (Database Issue): D1049-D1056, 2015.

19. Huang $J$ and Ling CX: Using AUC and accuracy in evaluating learning algorithms. IEEE Transact Know Data Eng 17: 299-310, 2005.

20. Ma S, Shah S, Bohnert HJ, Snyder M and Dinesh-kumar SP: Incorporating motif analysis into gene co-expression networks reveals novel modular expression pattern and new signaling pathways. PLoS Genet 9: e1003840, 2013.

21. Zhang B and Horvath S: A general framework for weighted gene co-expression network analysis. Stat Appl Genet Mol Biol 4: Article17, 2005.

22. Horvath S and Dong J: Geometric interpretation of gene coexpression network analysis. PLoS Comput Biol 4: e1000117, 2008.

23. Yip AM and Horvath S: Gene network interconnectedness and the generalized topological overlap measure. BMC Bioinformatics 8: 22,2007 .

24. Hauke J and Kossowski T: Comparison of values of pearson's and spearman's correlation coefficients on the same sets of data. Quaestiones Geographicae 30: 87-93, 2011.

25. Chua HN, Sung WK and Wong L: Exploiting indirect neighbours and topological weight to predict protein function from protein-protein interactions. Bioinformatics 22: 1623-1630, 2006

26. Weston AD and Hood L: Systems biology, proteomics, and the future of health care: Toward predictive, preventative, and personalized medicine. J Proteome Res 3: 179-196, 2004.

27. Peña-Castillo L, Tasan M, Myers CL, Lee H, Joshi T, Zhang C, Guan Y, Leone M, Pagnani A, Kim WK, et al: A critical assessment of Mus musculus gene function prediction using integrated genomic evidence. Genome Biol 9 (Suppl 1): S2, 2008.

28. Grice EA, Snitkin ES, Yockey LJ, Bermudez DM; NISC Comparative Sequencing Program, Liechty KW and Segre JA: Longitudinal shift in diabetic wound microbiota correlates with prolonged skin defense response. Proc Natl Acad Sci USA 107: 14799-14804, 2010.

29. Osborn O and Olefsky JM: The cellular and signaling networks linking the immune system and metabolism in disease. Nat Med 18: 363-374, 2012.

30. Finn OJ: Immuno-oncology: Understanding the function and dysfunction of the immune system in cancer. Ann Oncol 23 (Suppl 8): viii6-viii9, 2012.

31. Smith SE, Li J, Garbett K, Mirnics K and Patterson PH: Maternal immune activation alters fetal brain development through interleukin-6. J Neurosci 27: 10695-10702, 2007.

32. Fenzl SC, Hirsch T and Wolfbeis OS: Photonic crystals for chemical sensing and biosensing. Angew Chem Int Ed Engl 53: 3318-3335, 2014. 